\title{
Assessment of an augmented reality apparatus for the study of visually guided walking and obstacle crossing
}

\author{
Kamran Binaee $^{1} \cdot$ Gabriel J. Diaz $^{1}$ \\ Published online: 21 August 2018 \\ (C) Psychonomic Society, Inc. 2018
}

\begin{abstract}
To walk through the cluttered natural environment requires visually guided and anticipatory adjustments to gait in advance of upcoming obstacles. However, scientific investigation of visual contributions to obstacle crossing have historically been limited by the practical issues involved with the repeated presentation of multiple obstacles upon a ground plane. This study evaluates an approach in which the perception of a 3D obstacle is generated from 2D projection onto the ground plane with perspective correction based on the subject's motion-tracked head position. The perception of depth is further reinforced with the use of stereoscopic goggles. To evaluate the validity of this approach, behavior was compared between approaches to two types of obstacles in a blocked design: physical obstacles, and the augmented reality (AR) obstacles projected upon the ground plane. In addition, obstacle height, defined in units of leg length (LL), was varied on each trial $(0.15,0.25,0.35$ LL). Approaches to ended with collision on $0.8 \%$ of trials with physical obstacles per subject, and on $1.4 \%$ trials with AR obstacles. Collisions were signaled by auditory feedback. Linear changes in the height of both AR and physical obstacles produced linear changes in maximum step height, preserving a constant clearance magnitude across changes in obstacle height. However, for AR obstacles, approach speed was slower, the crossing step peaked higher above the obstacle, and there was greater clearance between the lead toe and the obstacle. These results suggest that subjects were more cautious when approaching and stepping over AR obstacles.
\end{abstract}

Keywords Gait · Obstacle crossing · Augmented reality · Vision

\section{Introduction}

When walking over uneven terrain, humans must make visually guided adjustments to gait in the advance of upcoming obstacles (Chou, Kaufman, Brey, \& Draganich, 2001; Matthis, Barton, \& Fajen, 2015; Patla \& Greig, 2006). Because this is inherently a visuo-motor task, research on the matter requires a ground plane that can be parametrically adjusted, perhaps by the addition, removal, or manipulation of physical obstacles between approaches. However, the need to frequently adjust physical obstacles is time consuming, places limitations on the types of practical manipulations one can implement, the number of obstacles that may be included within a single trial, and the number of trials that may be included within a single experiment. Furthermore, the approach offers little control over visual

Kamran Binaee

kb4000@rit.edu

1 Chester F. Carlson Center for Imaging Science, Rochester Institute of Technology, Rochester, NY, USA parameters of the stimulus such as contrast, or appearance. In this manuscript, we present a novel projector-based apparatus for the study of gait over computationally generated terrain with illusory height. This apparatus is considered a test-bed for the use of augmented reality (AR) technology, which allows computational imagery to be overlaid upon real-world imagery, typically through the use of transparent head-mounted displays.

The apparatus presented in this manuscript builds upon a long history of efforts to move beyond the limitations of standard desk-based psychophysics for the laboratory study of locomotion. For example, some have turned to the use of goggles that occlude approximately two steps of the subjects' lower visual field during the approach to and step over obstacles with height (Patla \& Greig, 2006; Rhea \& Rietdyk, 2007; Rietdyk \& Drifmeyer, 2010; Rietdyk \& Rhea, 2006). These goggles are affordable, lightweight, and effortless to implement.

Some of these issues have been addressed by the use of "smart" occluding glass, which have the additional advantage that they can be computationally triggered with very 
low latency, and high temporal precision (Mohagheghi, Moraes, \& Patla, 2004; Patla \& Greig, 2006). Remote activation of the occlusion is especially powerful when linked to a behavioral event, such as a step upon a pressure plate, or arrival of the foot or head at a predefined location.

A few studies have adopted the use of computationally generated targets/obstacles to gait that are projected upon the ground plane (Berg \& Mark, 2005; Matthis \& Fajen, 2013, 2014; Matthis et al., 2015). This approach is advantageous in that it allows for manipulations of visual information that are triggered by the position of the feet, head, or center of mass, or for manipulations of visual information that are triggered by events in the gait cycle (e.g., heel-strike, or toe-off). Because the projected stimulus is both computationally generated and projected, it affords investigators the ability to instantly vary the parameters of specific targets or obstacles, such as the availability of visual information, or information about the size or location. The system allows for behaviorally contingent manipulations, such as distant and gait-dependent rendering of targets and obstacles. The use of a computationally generated stimulus also facilitates the scaling of stimulus size to body dimensions, and stimulus onset to parameters of gait (e.g. step length). Because the targets are projected upon the real-world ground plane, the stimulus preserves accurate optic flow in the periphery, which has been found to play a role in the visual guidance of gait (Graci et al., 2009; Marigold \& Patla, 2008; Turano et al., 1999). The simultaneous use of motion-capture for real-time tracking of the subject's position during locomotion over the projected stimulus affords precise computational analysis of spatiotemporal data reflecting the recorded positions of the obstacle and walker within a common frame of reference. These many features enable researchers to address longstanding questions that could not be addressed through more conventional means.

The present study examines a modified version of the methodology of Matthis and Fajen (Matthis \& Fajen, 2013, 2014; Matthis et al., 2015) for the investigation of visually guided locomotion in a way that allows for the projection of augmented reality (AR) obstacles with illusory height (Fig. 1). The 3D percept is generated from a 2D projection onto the ground plane with perspective correction based on the subjects motion-tracked head position, consistent with a single-plane form of a virtual reality "cave" environment, (Cruz-Neira et al., 1993). The illusion of height in our study is further reinforced through the use of stereoscopic shutter glasses. Previously, stereo vision has been found to play a large role in visuomotor behavior during locomotion (Hayhoe, Gillam, Chajka, \& Vecellio, 2009). Furthermore, because the ground plane is aligned with the real-world ground plane, it retains the benefits of optic flow in the periphery, which has previously been found to play a role in navigation during human locomotion (Turano, Yu, Hao, \& Hicks, 2005). Collisions with the AR obstacle were signaled using auditory feedback.

The potential benefits of the proposed apparatus would be numerous, but only on the condition that the apparatus demonstrates ecological validity to the real-world context it intends to mimic. To determine the differences and similarities in gait behavior between the two conditions, the present study investigates the behavior of subjects as they approach and step over either physical or AR obstacles that systematically vary in height. We predict that if the AR apparatus proposed in this manuscript degrades the visual sources of information required for obstacle avoidance in the natural context, it should become apparent in less accurate scaling of foot height to the height of AR obstacles, differences in approach kinematics, and increased collision rates. In contrast, if the AR apparatus supports natural behavior, then visuo-motor strategies and resulting gait kinematics (e.g. the scaling of step height to obstacle height) should be invariant across obstacle type.

\section{Methods}

Stimuli were developed on a PC running Windows 7 on an Intel i7 processor, using the Vizard virtual reality toolkit version 5.2, by Worldviz (WorldViz LLC, Santa Barbara, CA, USA). The stimulus was rendered using an Nvidia Quadro 4000 (Nvidia Corporation, Santa Clara, CA, USA). A Quadro line card was chosen specifically for its ability to perform the Quad-buffering necessary for stereoscopic display (Hutchinson \& Kuester, 2004). This card was paired with an AMD Radeon R9 200 Series for rendering of the experimenter's desktop console (Advanced Micro Devices, Inc., Sunnyvale, CA, USA). The pairing of an NVidia Quadro with an AMD card was preferred to avoid known driver issues when pairing a Quadro with an NVidia GeForce line card in a single machine.

The Quadro 4000 graphics card was connected to a Volfoni RF transmitter hub, which is a synchronized pair of wireless Volfoni Edge3D stereoscopic glasses worn by the subject throughout the experiment (Volfoni Corporation, Paris; see Fig. 1). The stereoscopic glasses shuttered in synchrony with the alternating frames projected by a BenQ MW870UST ultra-short throw projector at $120 \mathrm{~Hz}(60 \mathrm{~Hz}$ per eye) at a resolution of $1280 \times 720$ pixels (Benq Corporation, Taipei, Taiwan). The projector was mounted to a tripod positioned $1.60 \mathrm{~m}$ off the walking axis, and at a height of $1.6 \mathrm{~m}$. The projector orientation was set using a bubble-level to ensure that it was at a right angle to the floor. Because this was an ultra short-throw projector, this 90 degree rotation created a rectified image upon the floor without the the need for additional software or hardware 


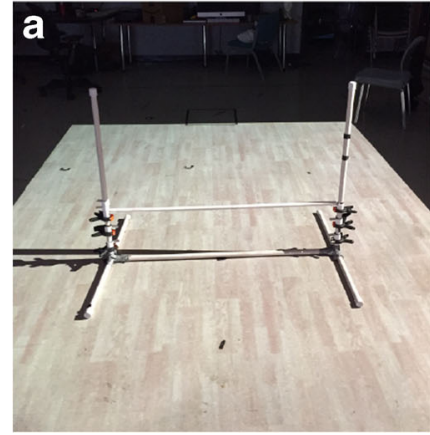

Fig. 1 a The physical obstacle lies inside the projection volume. b When viewed from the motion-tracked head position, stereoscopic imagery of the augmented reality obstacle projected upon the ground plane retains the perspective distortion experienced when viewing the physical obstacle from the same viewing position. c When the augmented reality obstacle is viewed away from the motion-tracked position, the projected 2D imagery appears distorted keystoning. The projected image occupied a space of 4.15 by $2.6 \mathrm{~m}$ on the laboratory floor.

Movements of the stereoscopic glasses and the subject's body were tracked by a 14 camera PhaseSpace X2 motion capture system running at $120 \mathrm{~Hz}$ (PhaseSpace, San Leandro, CA, USA). The system was used to measure the position and orientation of the stereoscopic glasses and the subject's feet. The foot was outfitted with four markers: one above the subject's big toe, one on the subject's heel, and two on the outer edge of the foot. In addition, the system monitored the position of four markers rigidly mounted to a small belt-mounted Plexiglas surface placed near the base of the subject's spine for the purpose of roughly approximating the subject's center of mass during locomotion.

The perspective correction was implemented using Vizard's vizcave module. This module creates an oblique (e.g. asymmetric) viewing frustum that extends from the location of the virtual camera to a predefined 2D plane within the virtual environment (i.e. the virtual ground plane). The size and position of this $2 \mathrm{D}$ plane was set to match the size and position of the laboratory floor illuminated by the projector. The virtual camera's position was updated with the changing position of the subject's motion-tracked head.

Scene luminance of the obstacle and surrounding environment (Fig. 1 was measured through the shutter glasses from a standing distance of $1 \mathrm{~m}$, and an eye-height of $1.5 \mathrm{~m}$. The luminance of AR obstacle ranged from 3.3 $\mathrm{cd} / \mathrm{m}^{2}$ in the brightest regions to $0.4 \mathrm{~cd} / \mathrm{m}^{2}$ in the darker, shaded regions. The luminance of the physical obstacle was $2.9 \mathrm{~cd} / \mathrm{m}^{2}$, the surrounding texture of the parquet floor texture within the projection volume was $1.2 \mathrm{~cd} / \mathrm{m}^{2}$, and the luminance of the lab floor surrounding the projected volume $0.15 \mathrm{~cd} / \mathrm{m}^{2}$. There were no noticeable issues related to glare when traversing the walking axis; although specular reflections were visible on the floor between the projector and the observer's viewpoint, these reflections were not aligned with the subject's path, and did not obscure the obstacle during the approach.

The end-to-end latency of the system, defined as the duration between the occurrence of a physical movement of a tracked rigid body and the time that this image produced a subsequent change in rendered imagery, is estimated to be slightly above $30 \mathrm{~ms}$. This estimate is based on the measured latency of $27 \mathrm{~ms}$ when the image was displayed on a CRT monitor, and the assumption of additional latency that may be attributed to the use of a DLP projector.

Experimental data and motion capture data capture data were exported from Vizard for alignment and analysis in Matlab R2015a.

\section{Participants}

Fourteen undergraduate students from the Rochester Institute of Technology (RIT) volunteered to participate in the study $(M=9, F=5)$. Ages ranged from $18-26(M=$ 21.6, $\mathrm{SD}=2.6)$. Subjects reported that they had normal or corrected-to-normal vision and did not have any visual or motor impairments. The protocol was approved by the Institutional Review Board at RIT and all subjects gave informed consent prior to participation. The number of participants was chosen to match or exceed the numbers used in published studies on obstacle crossing of similar design (Chou \& Draganich, 1998; Chou et al., 2001; Mohagheghi et al., 2004; Patla \& Greig, 2006; Patla \& Rietdyk, 1993; Rhea \& Rietdyk, 2007; Timmis \& Buckley, 2012).

\section{Experimental design}

Subjects arrived at the lab wearing comfortable walking shoes or sneakers. The experiment involved stepping over an obstacle placed or projected within a $5.5-\mathrm{m}$ walking path 
over an otherwise flat ground plane (Fig. 2). The obstacle distance from the start box was randomly selected from the range of 2.7 through $3.3 \mathrm{~m}$. Obstacle type (AR or physical) was varied between four blocks (two AR, two physical). To prevent an asymmetric influence of fatigue or experience on a single-condition, the obstacle type encountered on the first block was counter-balanced between individuals (seven AR first, seven physical first). On each trial, obstacle height was randomly selected from three possible heights. To reduce between subject differences due to differences in body size, obstacle height was scaled to the leg length (LL) of the shoed participant. Leg length was measured prior to data collection using a tape measure that extended from the floor, to the location of hip joint (i.e. acetabulofemoral joint), which was estimated during joint rotation as the standing participant lifted his or her foot of the ground. Mean leg length was $0.92 \mathrm{~cm}(\mathrm{SEM}=0.02)$. The height of the obstacle was selected from one of three possible heights: $0.15 \mathrm{LL}, 0.25 \mathrm{LL}$, or $0.35 \mathrm{LL}$. These values were entered into the Vizard stimulus generation application, and used when rendering the AR obstacles. Each height was repeated 12 times per block, for a total of 36 trials within a block. In summary, the experimental design involved 12 trials per three levels of obstacle height within each of four blocks which differed by obstacle type (physical vs. AR). This yielded a total of 144 trials per participant. The duration between the start of the first trial and the end of data collection was approximately $45 \mathrm{~min}$.

\section{Obstacle appearance and dimensions}

The physical obstacle was a PCB agility gait more typically used for dog agility tests (Fig. 1). Obstacle width was $1.24 \mathrm{~ms}$, depth was $0.87 \mathrm{~m}$, and the radius of each component bar was approximately $1 \mathrm{~cm}$. The crossbar was not affixed to the uprights, but rested upon plastic cups to minimize the risk of tripping upon collision. To facilitate rapid adjustment of the crossbar to the appropriate height on physical trials, three pairs of cross-bar supporting cups were affixed to the gate's uprights at the predetermined obstacle heights (in units of leg length) prior to experimentation. The AR obstacle model was created using white cylinder primitives in Vizard. The obstacle was rendered using a white diffuse material, and the position, orientation, length, and radius of the cylinders were matched to the physical obstacle. Crossbar height was adjusted automatically within Vizard on a trial-by-trial basis. When scaled to the leg lengths of our participants mean obstacle heights were 13.3 $\mathrm{cm}, 22.2 \mathrm{~cm}$, and $31 \mathrm{~cm}$ tall.

\section{Procedure}

Subjects were instructed to walk at a comfortable pace from the starting box to the ending box, and to try and maintain a constant pace throughout the experiment. The subject began each trial by standing in the designated starting box. On trials with AR obstacles, entry to the box would trigger the projection of the obstacle. The subject was required to wait for $2 \mathrm{~s}$ within the box before departing, for the purpose of providing a preview of the obstacle prior to the initiation of movement (as would be the case in the physical obstacle condition). Departure from the box prior to this 2-s period would cause the AR obstacle to disappear. After the $2 \mathrm{~s} \mathrm{had}$ passed, an auditory tone would signal the subject that it was an appropriate time to begin walking.

On physical trials, entry into the start box would trigger the appearance of visual indicators used to indicate to the experimenter where the physical obstacle should be placed, and the intended obstacle height. These indicators included a thin horizontal line projected upon the groundplane. In addition, an indication of whether the randomly selected obstacle height was projected to a location on the

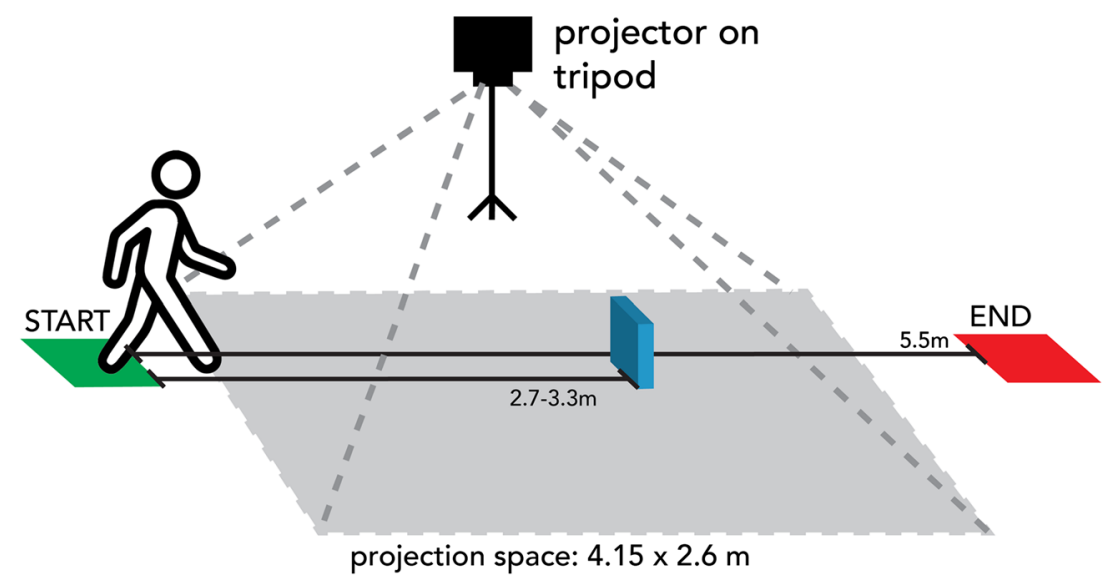

Fig. 2 The laboratory configuration. Subjects wearing stereo shutter glasses walked a distance of $5.5 \mathrm{~m}$ between the start and end box while avoiding a physical or AR obstacle placed/projected within their path 
floor several meters from the obstacle in the form of text: "short", "medium", or "tall". Subsequently, the experimenters shifted the position of the agility gate to the location of the line, and moved the detachable crossbar to one of three pairs of supporting cups previously affixed to the verticals at the appropriate height. The trial ended six seconds following the departure of a foot from the starting box.

The subject would receive auditory feedback if the motion-tracked feet collided with either the physical or AR obstacle. Collisions were monitored at $120 \mathrm{~Hz}$ using Vizard's native physics engine by placing an invisible rectangular object upon the ground plane at the location of the obstacle, scaled to obstacle size. Latency between collision and auditory feedback was approximately $60 \mathrm{~ms}$ when measured using a high-speed video camera operating at $120 \mathrm{~Hz}$.

\section{Statistical analyses}

Unless stated otherwise, significance tests were conducted with a two-way repeated measures ANOVA with an alpha of 0.05. Mauchly's test was applied to test for sphericity, and the Greenhouse-Geisser method was applied in the case that the assumption of sphericity had been violated. Although the use of multiple pairwise comparisons may run the risk of increased Type 1 error rate, correction through the traditional means would increase the possibility of Type 2 error, and lead to the false interpretation that behavior between the two conditions is similar (when in fact, it is not). We have opted for the more conservative approach: not to adjust for Type 1 error in response to the presence of multiple comparisons.

\section{Results}

\section{Collisions}

When approaching and stepping over a physical obstacle, subjects collided with the obstacle a total of eight times, and an average of $0.8 \%$ trials per subject $(\mathrm{SEM}=0.1 \%)$. The lead foot collided with an average of $0.3 \%$ of short physical obstacles ( $\mathrm{SEM}=0.3 \%, \mathrm{~N}=1$ trials), and $0.9 \%$ of tall physical obstacles ( $\mathrm{SEM}=0.6 \%, \mathrm{~N}=3$ trials). There were no collisions between the lead foot and the medium physical obstacles. The trail foot collided on an average with 0.6 short physical obstacles $(\mathrm{SEM}=0.6 \%, \mathrm{~N}=2$ trials), $0.3 \%$ of medium physical obstacles $(\mathrm{SEM}=0.3 \%, \mathrm{~N}=1$ trials), and $0.3 \%$ of tall physical obstacles (SEM $=0.3 \%$, $\mathrm{N}=1$ trials). When approaching and stepping over an AR obstacle, subjects collided with the obstacle a total of 14 times, and on an average of $1.4 \%$ trials per subject (SEM $=0.5 \%$ ). Although there were no collisions between either foot and the short AR obstacle, the lead foot collided with an average of $0.6 \%$ of medium AR obstacles (SEM $=0.49$ trials). The trail foot collided on an average with 0.3(SEM $=0.3 \%, \mathrm{~N}=1$ trials), and $0.6 \%$ of tall AR obstacles (SEM $=0.6 \%, \mathrm{~N}=2$ trials). Closer inspection reveals that of the nine collisions between the lead foot and the tallest obstacle, five can be attributed to a single participant.

\section{Approach velocity}

The forward velocity of the subject's center of mass (COM) for the approach phase is shown in (Fig. 3). Subjects accelerated early in the approach until reaching peak forward velocity at step N-2, two steps prior to arrival at the obstacle (steps N-4 to N-1 in Fig. 3).

Separate ANOVAs were run within each step, and the results are reported in Table 1 . There were no interaction effects between obstacle height and type for any step during the approach. There was a main effect for obstacle type on steps N-2, for the step on which the lead foot crossed over the obstacle (i.e. "lead over" in Fig. 3), and for the step on which the trailing foot crossed over the obstacle (i.e., "trail over"). During step N-1, and the subsequent two steps on which the lead foot and trail foot crossed over the obstacle (lead over and trail over in Fig. 3), there was a decrease of COM velocity in the forward direction. A significant

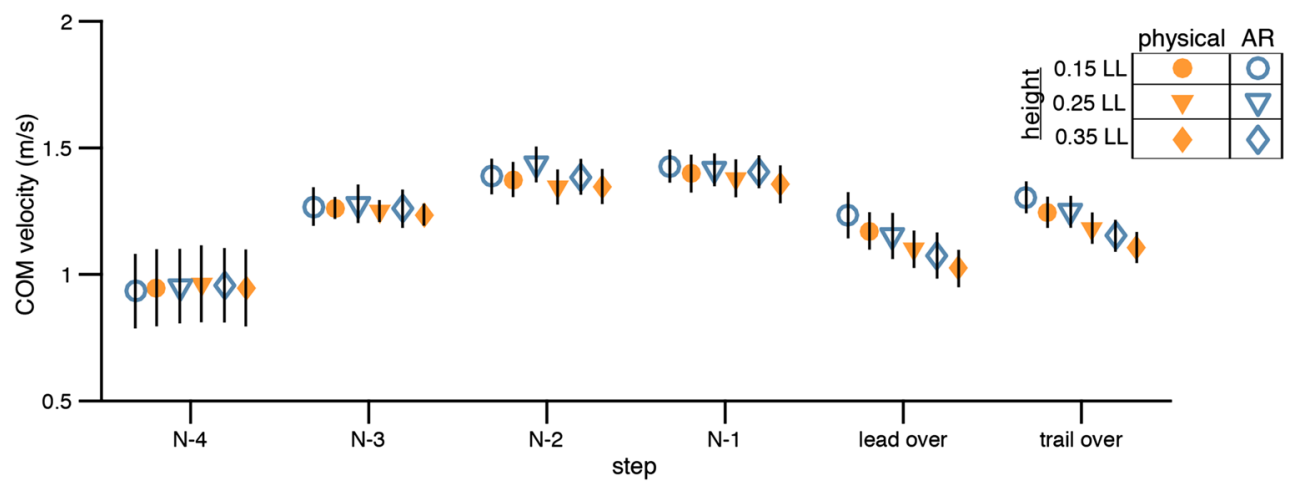

Fig. 3 Approach velocity for physical and AR obstacles of different heights. Error bars represent 95\% confidence intervals reflecting within-subject variability 
Table 1 Statistical tests for forward velocity of the center of mass

\begin{tabular}{lllrl}
\hline Step & IV & F-ratio & $p$ value & $\eta_{p}^{2}$ \\
\hline N-4 & Type & $\mathrm{F}(1,12)=0.44$ & 0.52 & 0.04 \\
N-4 & Height & $\mathrm{F}(2,24)=0.49$ & 0.62 & 0.04 \\
N-4 & Type*Height & $\mathrm{F}(1,13)=2.12$ & 0.17 & 0.15 \\
N-3 & Type & $\mathrm{F}(1,13)=1.14$ & 0.30 & 0.08 \\
N-3 & Height & $\mathrm{F}(2,26)=2.10$ & 0.14 & 0.14 \\
N-3 & Type*Height & $\mathrm{F}(2,26)=1.51$ & 0.24 & 0.10 \\
N-2 & Type & $\mathrm{F}(1,13)=5.92$ & 0.03 & 0.31 \\
N-2 & Height & $\mathrm{F}(1,16)=2.78$ & 0.11 & 0.18 \\
N-2 & Type*Height & $\mathrm{F}(1,16)=3.06$ & 0.09 & 0.19 \\
N-1 & Type & $\mathrm{F}(1,13)=3.58$ & 0.08 & 0.22 \\
N-1 & Height & $\mathrm{F}(2,26)=4.57$ & 0.02 & 0.26 \\
N-1 & Type*Height & $\mathrm{F}(2,26)=0.90$ & 0.42 & 0.06 \\
lead over & Type & $\mathrm{F}(1,13)=6.28$ & 0.03 & 0.33 \\
lead over & Height & $\mathrm{F}(1,14)=32.40$ & $<0.01$ & 0.71 \\
lead over & Type*Height & $\mathrm{F}(2,26)=0.10$ & 0.90 & 0.01 \\
trail over & Type & $\mathrm{F}(1,13)=12.24$ & $<0.01$ & 0.48 \\
trail over & Height & $\mathrm{F}(1,14)=28.73$ & $<0.01$ & 0.69 \\
trail over & Type*Height & $\mathrm{F}(2,26)=0.35$ & 0.71 & 0.03 \\
\hline & & & &
\end{tabular}

main effect of obstacle height indicates that the magnitude of the slowdown was greater for taller obstacles. There was a significant effect of obstacle type on approach speed for step N-2, but not for the other steps.

\section{Foot placement}

The interaction of obstacle height and type on placement of the feet was not significant (lead foot: $F(2.00,26.00$ ) $=0.21, p=0.81, \eta_{\mathrm{p}}^{2}=0.02$, trail foot: $\mathrm{F}(2.00,26.00)$ $\left.=0.19, p=0.83, \eta_{\mathrm{p}}^{2}=0.01\right)$. Despite the trial-by-trial changes in obstacle height, there was no significant effect of height on foot placement prior to the step over (Fig. 4; (lead: $\mathrm{F}(2.00,26.00)=0.98, p=0.39, \eta_{\mathrm{p}}^{2}=0.07$, trail: $\left.\mathrm{F}(2.00,26.00)=0.03, p=0.97, \eta_{\mathrm{p}}^{2}=0.00\right)$. Obstacle type had marginally significant effects on placement of the lead and trailing foot in front of the obstacle (lead: $\mathrm{F}(1.00,13.00)$ $=4.53, p=0.05, \eta_{\mathrm{p}}^{2}=0.26$, trail: $\mathrm{F}(1.00,13.00)=4.74, p$ $=0.05, \eta_{\mathrm{p}}^{2}=0.27$ ).

\section{Obstacle crossing}

Maximum step height and foot-obstacle distance for the lead and trail foot is shown in Fig. 5. No significant interactions of obstacle type or height were found for either maximum step height or clearance between the foot and obstacle (lead foot: $\mathrm{F}(1.22,15.84)=0.10, p=0.81, \eta_{\mathrm{p}}^{2}=0.01$, trail foot: $\left.\mathrm{F}(2.00,26.00)=1.42, p=0.26, \eta_{\mathrm{p}}^{2}=0.10\right)$.

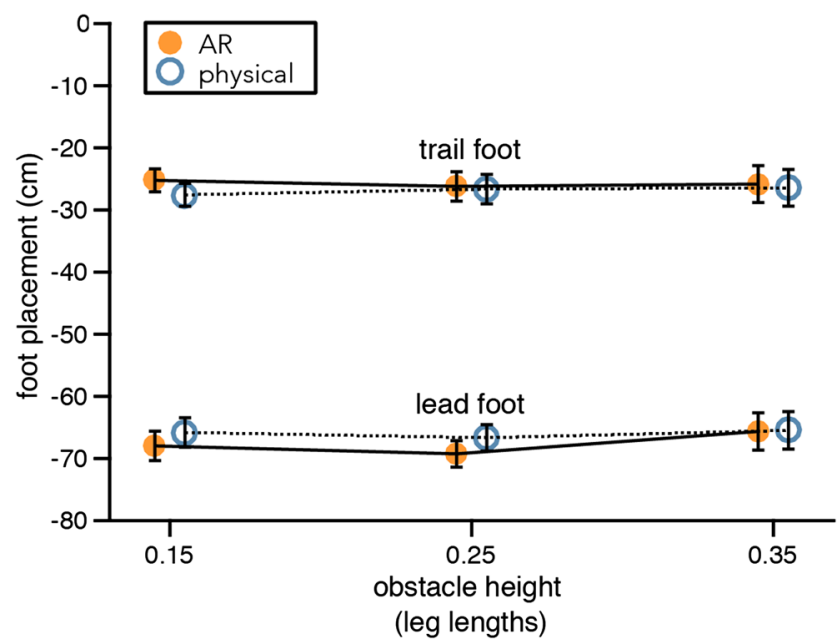

Fig. 4 Placement of the trail foot and lead foot in front of the obstacle prior to the step over. Error bars represent 95\% confidence intervals reflecting within-subject variability

The height of the crossing steps increased with obstacle height (lead foot: $\mathrm{F}(1.10,14.32)=255.63, p<0.01$, $\eta_{\mathrm{p}}^{2}=0.95$, trail foot: $\mathrm{F}(1.17,15.17)=306.87, p<0.01$, $\eta_{\mathrm{p}}^{2}=0.96$ ), as was necessary to avoid collisions with taller obstacles. The scaling of step height to obstacle height was linear, and brought about a constant minimum clearance distance between the lead foot and the obstacle across changes in obstacle height (lead foot: $\mathrm{F}(1.16,15.12)=0.41, p=$ $0.56, \eta_{\mathrm{p}}^{2}=0.03$, trail foot: $\mathrm{F}(1.29,16.81)=1.25, p=0.29$, $\left.\eta_{\mathrm{p}}^{2}=0.09\right)$.

There was an effect of obstacle type on maximum step height for the lead foot $(\mathrm{F}(1.00,13.00)=13.20, p<0.01$, $\left.\eta_{\mathrm{p}}^{2}=0.50\right)$, and a significant main effect on clearance between the toe marker and the top of the obstacle at the time the toe passed its front face $(\mathrm{F}(1.00,13.00)=7.25$, $p=0.02, \eta_{\mathrm{p}}^{2}=0.36$ ). These effects were not visible in the trailing foot, which demonstrated no significant effects of obstacle type on maximum step height $(\mathrm{F}(1.00,13.00)=$ $\left.0.34, p=0.57, \eta_{\mathrm{p}}^{2}=0.03\right)$ or clearance $(\mathrm{F}(1.00,13.00)=$ $\left.0.26, p=0.62, \eta_{\mathrm{p}}^{2}=0.02\right)$.

\section{General discussion}

This study tested the suitability of an augmented reality apparatus for the study of visually guided walking behavior. This mean collision rate of $0.8 \%$ for physical and $1.5 \%$ for AR obstacles is comparable to the values reported by other studies (Chou et al., 2001; Mohagheghi et al., 2004; Heijnen et al., 2012; Rhea \& Rietdyk, 2007), which range from no collisions when the maximum obstacle height was approximately $25 \mathrm{~cm}$ (Chou et al., 2001) to $1.25 \%$ collisions for a study in which maximum obstacle height was approximately $30 \mathrm{~cm}$ (Mohagheghi et al., 2004). 

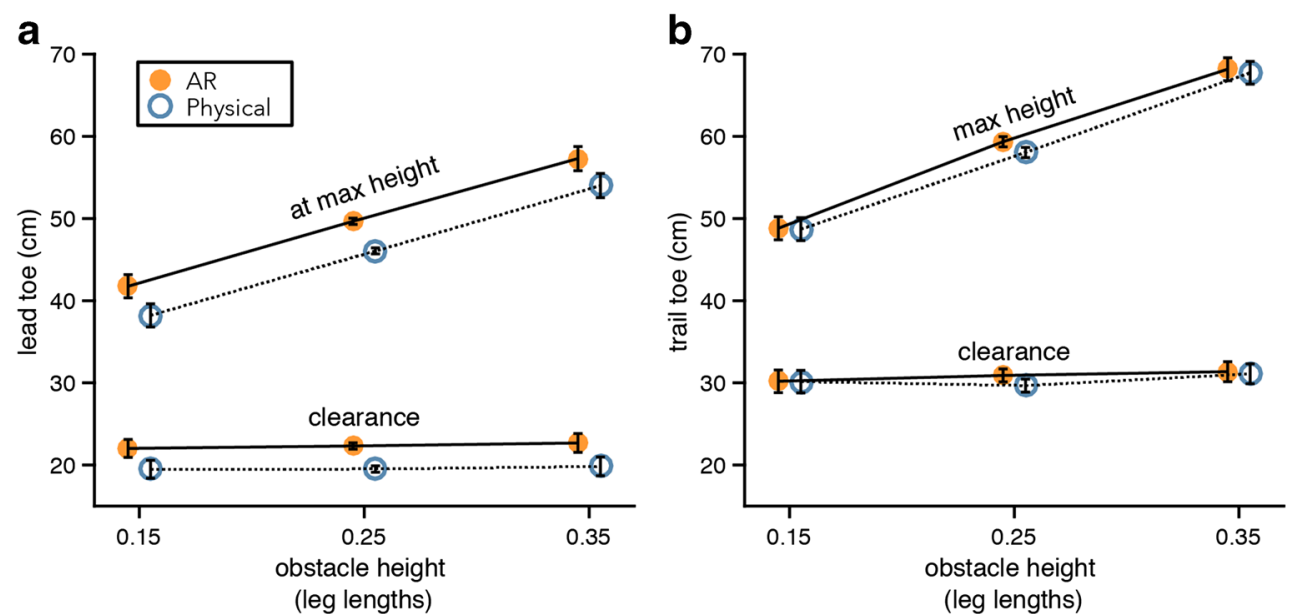

Fig. 5 Maximum step height and minimum clearance between the toe and obstacle on the step over the obstacle for both the a toe of the lead foot, and $\mathbf{b}$ the toe of the trailing foot. Error bars represent $95 \%$ confidence intervals re ecting within subject variability

Whether approaching physical or AR obstacles, subjects scaled their behavior to changes in obstacle height. In both conditions, subjects increased their velocity early in the approach, only to slow down in the final stages of the approach by a magnitude proportional to obstacle height (Fig. 3). Presumably, this decrease in forward velocity for taller obstacles was necessary for the redirection of COM velocity from the forward direction to the vertical direction (Chou et al., 2001). Consistent with previous work on the crossing of physical obstacles (Chou et al., 2001; Patla \& Greig, 2006), the distance of foot placement from the obstacle prior to the step over was constant across changes in obstacle height (Fig. 4). The height of the steps over the obstacle were scaled to obstacle height, and as a result the clearance between the toe marker and the front face of the obstacle remained invariant across changes in height for both physical and AR obstacles (Fig. 5). The observed range of clearance distances between the feet and the obstacle observed in the present study are within the range of previously published reports conducted in the natural environment using a similar style obstacle, and range of heights (Rietdyk $\&$ Rhea, 2011). This suggests that subjects were able to accurately scale the height of the crossing steps to changes in obstacle height for both AR and physical obstacles, and that overall behavior was consistent with previous reports.

Despite the subject's ability to perform the task, and qualitatively similar adjustments in response to changes in obstacle height, there were indications of increased uncertainty about obstacle height, and possibly position along the ground plane. The final steps of the subject's approach were slower when approaching an AR obstacle than when approaching a physical obstacle (steps N-2, lead over, and trail over in Fig. 3). There was also a marginally significant effect of obstacle type on placement of the lead and trailing feet prior to the step over (Fig. 4). Because prior work suggests that foot placement is invariant to changes in obstacle height (Chou et al., 2001; Patla \& Greig, 2006), and obstacle depth did not vary, this difference can tentatively be attributed to uncertainty in the position at which the obstacle contacts the ground. Kinematics of the step over also indicate some uncertainty in obstacle height. In both conditions, subjects modified maximum height of the crossing steps in a manner that accounted for changes in obstacle height. However, there was an overall increase in maximum step height for AR obstacles relative to physical obstacles. This produced a slightly greater distance between the toe marker and the obstacle as it passed over its front face.

\section{Current limitations and potential improvements}

Although the system adopted in this study elicited behavior that was appropriately scaled to obstacle height, there are clear paths to improvement. For example, the use of active shutter glasses dropped luminance of the AR obstacles from $10 \mathrm{~cd} / \mathrm{m}^{2}$ when viewed without the glasses to 3.3 $\mathrm{cd} / \mathrm{m}^{2}$. To improve brightness levels, one could replace the active shutter glasses with passive (e.g. polarized) 3D lenses, which are known to have a less dramatic effect upon perceived brightness, but which require that a polarizing modulator is incorporated into the projection system. Due to the use of projectors, the obstacle and the observer's body will cast shadows on the occluded portions of the projected image (Fig. 1a). In the present study, it was reasonable to position the projector orthogonally to the subject's walking path, resulting in shadows cast to the observer's side as they approached the obstacle. Although these shadows did not obscure the obstacle itself, their presence may be distracting or otherwise problematic in other experimental designs. To mitigate the effects of shadows, one could incorporate additional projectors that display the same content from other positions, so that occlusion would 
result in a drop in contrast, but not a total lost of imagery. However, to accurately align overlapping imagery from multiple projectors requires additional software for spatial calibration and color correction (e.g. Vioso AnyBlend; Vioso GmbH, Dusseldorf Germany).

In our arrangement the placement of the projector ensured that specular reflections visible on the floor between the projector and the observer's viewpoint would never fall upon the image of the obstacle. A person concerned about the presence of specular reflections/glare could prepare their projection surface with matte paint.

Finally, although the AR obstacles used in our paradigm lack the tactile or haptic feedback thought to be important to the trial-and-error adaptation to obstacle dimensions (Heijnen \& Rietdyk, 2018). However, it is likely the lowlatency auditory feedback provided upon collision will mitigate this limitation.

\section{Comparison to conventional augmented reality and virtual reality displays}

This study provides some insight into the potential role of emerging technologies related to virtual and augmented reality displays for the study of visually guided walking. A beneficial property of the projection method is that, because the augmented reality surface is overlaid upon the real world environment, the subject's field of view of the natural environment is unrestricted (although a portion is blocked by the thin frame of the shutter glasses), and the augmented reality content may overlay a large area of the floor (4.15 $\times 2.6 \mathrm{~m}$; Fig. 2). Although contemporary augmented reality systems are also able to combine computational imagery with the real-world scene, and have the added benefit of being fully mobile and battery operated, it is notable that the computational imagery may only be projected to narrow portion of the observer's field of view. For example, the Microsoft Hololens (Microsoft Corporation, Redmond, WA, USA), is only able to present AR content within a region that subtends 30 degrees along the horizontal, and 17 degrees along the vertical. In contrast, the field of view in contemporary virtual reality HMD are in the region of $100^{\circ}$ along the horizontal axis (e.g., Oculus Rift, or HTC Vive). However, because these systems are unable to combine the virtual and augmented imagery, the user may not have visual feedback concerning leg or foot position during locomotion. One might attempt to resolve this through the use of motion tracking to animate a virtual avatar visible inside the simulated environment. In practice, this is surprisingly difficult; even small misalignments between the optical axis defined by the HMD and the viewing frustum within the rendering pipeline yield a visual effect similar to those experienced when using optical prism glasses, whereby there is an artificial visual offset between the visual location of the virtual foot and the true location of the subject's feet relative to his or her head. This offset would also result in a similar misalignment of the virtual and real ground planes in terms of both position and orientation. Although these effects are likely to influence an observer's gait behavior, there has been no systematic investigation that we are aware of.

\section{Conclusions}

Although augmented reality presents a convenient new method for the study of visual contributions to the control of gait, the projector-based AR apparatus utilized in this study introduced a constant bias towards more cautious behavior when subjects approached and stepped over AR obstacles. Despite this heightened level of caution, the relative change in behavior (e.g., step height) when negotiating obstacles of different heights was the same for physical and augmented reality obstacles. Thus, the apparatus is able to produce realistic adaptations to changing properties of the obstacle. When considering the suitability of this projector AR apparatus, it is best practice that any potentially negative impact of these issues are taken into account on a case-bybase basis.

\section{References}

Berg, W. P., \& Mark, L. S. (2005). Information for step length adjustment in running. Human Movement Science, 24(4), 496531. https://doi.org/10.1016/j.humov.2005.07.002

Chou, L. S., \& Draganich, L. F. (1998). Placing the trailing foot closer to an obstacle reduces flexion of the hip, knee, and ankle to increase the risk of tripping. Journal of Biomechanics, 31(8), 685691. https://doi.org/10.1016/S0021-9290(98)00081-5

Chou, L. S., Kaufman, K. R., Brey, R. H., \& Draganich, L. F. (2001). Motion of the whole body's center of mass when stepping over obstacles of different heights. Gait \& Posture, 13(1), 17-26. https://doi.org/10.1016/S0966-6362(00)00087-4. http:// linkinghub.elsevier.com/retrieve/pii/S0966636200000874

Cruz-Neira, C., Sandin, D., \& DeFanti, T. (1993). Surroundscreen projection-based vir- 414 tual reality: the design and implementation of the CAVE. . . . of the 20Th Annual 415 Conference on ....pp 135-142.

Graci, V., Elliott, D. B., \& Buckley, J. G. (2009). Peripheral visual cues aKect minimum-foot-clearance during overground locomotion. Gait and Posture, 30(3), 370-374. https://doi.org/10.1016/j.gaitpost.2009.06.011

Hayhoe, M., Gillam, B., Chajka, K., \& Vecellio, E. (2009). The role of binocular vision in walking. Visual Neuroscience, 26(01), 73. https://doi.org/10.1017/S0952523808080838. http:// www.journals.cambridge.org/abstract_S0952523808080838

Heijnen, M. J., \& Rietdyk, S. (2018). Failures in adaptive locomotion: trial-and-error exploration to determine adequate foot elevation over obstacles. Experimental Brain Research, 236(1), 187-194. https://doi.org/10.1007/s00221-017-5117-y

Heijnen, M. J. H., Muir, B. C., \& Rietdyk, S. (2012). Factors leading to obstacle contact during adaptive locomotion. Experimental Brain Research, 223(2), 219-231. https://doi.org/10.1007/s00221012-3253-y 
Hutchinson, T. C., \& Kuester, F. (2004). Hardware architecture for a visualization classroom: Vizclass. Computer Applications in Engineering Education, 12(4), 232-241.

Matthis, J. S., Barton, S. L., \& Fajen, B. R. (2015). The biomechanics of walking shape the use of visual information during locomotion over complex terrain. Journal of Vision, 15(3), 10. https://doi.org/10.1167/15.3.10.doi

Matthis, J. S., \& Fajen, B. R. (2013). Humans exploit the biomechanics of bipedal gait during visually guided walking over complex terrain. Proceedings of the Royal Society B: Biological Sciences, 280(1762), 20130,700. https://doi.org/10.1098/rspb.2013. 0700

Matthis, J. S., \& Fajen, B. R. (2014). Visual control of foot placement when walking over complex terrain. Journal of Experimental Psychology: Human Perception and Performance, 40(1), 10615. https://doi.org/10.1037/a0033101. http://www.ncbi.nlm.nih. gov/pubmed/23750964.

Mohagheghi, A. A., Moraes, R., \& Patla, A. E. (2004). The effects of distant and on-line visual information on the control of approach phase and step over an obstacle during locomotion. Experimental Brain Research, 155(4), 459-468. https://doi.org/10.1007/s00221003-1751-7

Marigold, D. S., \& Patla, A. E. (2008). Visual information from the lower visual Leld is important for walking across multisurface terrain. Experimental Brain Research, 188(1), 23-31. https://doi.org/10.1007/s00221-008-1335-7

Patla, A., \& Rietdyk, S. (1993). Visual control of limb trajectory over obstacles during locomotion: Effect of obstacle height and width. Gait and Posture, 1(1), 45-60. https://doi.org/10.1016/0966-6362 (93) $90042-Y$

Patla, A. E., \& Greig, M. (2006). Any way you look at it, successful obstacle negotiation needs visually guided on-line foot placement regulation during the approach phase. Neuroscience Letters, 397(1-2), 110-114. https://doi.org/10.1016/j.neulet.2005.12.016

Rhea, C. K., \& Rietdyk, S. (2007). Visual exteroceptive information provided during obstacle crossing did not modify the lower limb trajectory. Neuroscience Letters, 418(1), 60-65. https://doi.org/10.1016/j.neulet.2007.02.063

Rietdyk, S., \& Drifmeyer, J. E. (2010). The rough-terrain problem: Accurate foot targeting as a function of visual information regarding target location. Journal of Motor Behavior, 42(1), 37-48. https://doi.org/10.1080/00222890903303309. http://www. ncbi.nlm.nih.gov/pubmed/20018585

Rietdyk, S., \& Rhea, C. K. (2006). Control of adaptive locomotion: Effect of visual obstruction and visual cues in the environment. Experimental Brain Research, 169(2), 272-8. https://doi.org/10.1007/s00221-005-0345-y. http://www.ncbi.nlm. nih.gov/pubmed/16421728

Rietdyk, S., \& Rhea, C. K. (2011). The effect of the visual characteristics of obstacles on risk of tripping and gait parameters during locomotion. Ophthalmic and Physiological Optics, 31(3), 302-310. https://doi.org/10.1111/j.1475-1313.2011.00837.x

Timmis, M. A., \& Buckley, J. G. (2012). Obstacle crossing during locomotion: Visual exproprioceptive information is used in an online mode to update foot placement before the obstacle but not swing trajectory over it. Gait and Posture, 36(1), 160-162. https://doi.org/10.1016/j.gaitpost.2012.02.008

Turano, K. A., Rubin, G. S., \& Quigley, H. A. (1999). Mobility performance in glaucoma. Investigative Ophthalmology and Visual Science, 40(12), 2803-2809.

Turano, K. A., Yu, D., Hao, L., \& Hicks, J. C. (2005). Optic-flow and egocentric-direction strategies in walking: Central vs peripheral visual field. Vision Research, 45(25-26), 3117-3132. https://doi.org/10.1016/j.visres.2005.06.017. http:// linkinghub.elsevier.com/retrieve/pii/S0042698905003020 